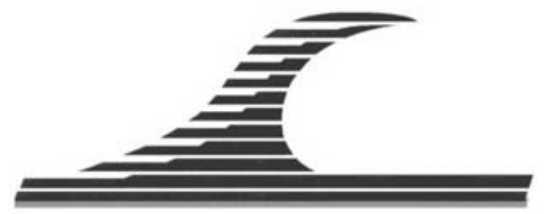

Revue Paralia, Volume 3 (2010) pp 7.1-7.12

Mots-clés : Dragages, Turbidité, Ecosystème récifal, Base de données interopérables, Suivi environnemental, Développement durable, Nouvelle-Calédonie

(C) Editions Paralia CFL

\title{
Le suivi de la turbidité associée au dragage de Vavouto à proximité des sites néocalédoniens classés par l'UNESCO
}

\author{
Michel ALLENBACH ${ }^{1}$, Morgan MANGEAS ${ }^{2}$, TOURAIVANE ${ }^{1}$
}

1. Université de la Nouvelle-Calédonie, PPME, EA 3325, BPR4, 98851 Nouméa Cedex. allenbach@univ-nc.nc; touraivane@univ-nc.nc

2. IRD Centre de Nouméa, US Espace, BP A5, 98848 Nouméa Cedex.

morgan.mangeas@ird.noumea.nc

\section{Résumé :}

La construction d'une usine métallurgique de nickel, de dimension mondiale nécessite l'ouverture d'un important chenal de navigation (4500 m de longueur et $7300000 \mathrm{~m}^{3}$ de déblais) dans le lagon nord-ouest néo-calédonien, à proximité immédiate de zones coralliennes classées au patrimoine mondial en raison de leur biodiversité exceptionnelle. L'enjeu est essentiel : contribuer au rééquilibrage économique inscrit dans les lois, dans le respect d'un environnement fragile et des pratiques sociétales des populations riveraines. La communication présente le plan de suivi environnemental, intégrant un réseau de suivi en temps réel de la turbidité et des paramètres physicochimiques de l'eau et des sédiments, ainsi qu'un important volet biologique. Sont ensuite exposés les résultats des contrôles environnementaux réalisés et l'intérêt d’une démarche originale pour assurer leur restitution aux populations autochtones : la création du CEK (comité environnemental du Koniambo). La dernière partie traite de l'intérêt de l'utilisation de bases des données interopérables : elles facilitent la communication en interne et en externe et rendent plus aisées les méthodes de contrôle à mettre en œuvre.

Soumis le 30 octobre 2010, accepté le 5 novembre 2010, en ligne le 20 décembre 2010.

La seule version examinée est celle écrite en français. La ou les autres versions n'étant pas examinées par le comité de rédaction de la revue, sont donc publiées sous l'entière responsabilité du ou des auteurs.

A TRANSLATED VERSION IN ENGLISH IS AVAILABLE ONLINE

Pour citer cet article :

ALLENBACH M., MANGEAS M., TOURAIVANE (2010). Le suivi de la turbidité associée au dragage de Vavouto à proximité des sites néocalédoniens classés par l'UNESCO. Revue Paralia, Vol. 3, pp 7.1-7.12. 


\section{Introduction. Présentation générale du "Projet Koniambo"}

Sur la côte nord-ouest de la Grande Terre néo-calédonienne, dans le Pacifique sudouest, la construction d'une usine métallurgique de transformation du minerai de nickel est en phase opérationnelle et devrait se terminer en 2013. Le chantier, de dimension mondiale, impacte la zone "VKP", du nom des trois communes (Voh, Koné, Poimbout) sur lesquelles sont implantées l'essentiel des infrastructures directes et indirectes d'un programme industriel clef de "l’Accord de Nouméa" : le projet Koniambo, du nom du massif minier qui va être mis en exploitation pour alimenter l'usine. Les infrastructures à créer (usine, base-vie, centrale thermique, site minier) génèrent des besoins significatifs en matières premières (charbon, hydrocarbures, modules préfabriqués de l'usine) à importer, et la nécessité, en retour, d'exporter le produit fini (ferro-nickel). Dans le contexte insulaire océanien, la voie maritime s’imposait pour ces transports et la décision a été prise de construire un port. Le site portuaire choisi (Vavouto) nécessite l'ouverture d'un chenal de navigation, pour permettre l'accès sécuritaire au site à des navires pouvant atteindre 50000 TPL.

\section{Descriptif rapide des travaux de dragage}

\subsection{Données générales sur les travaux}

Confiés à la société "Jan de Nul", l'une des "majors" du dragage mondial, les travaux ont débuté en août 2008 et doivent se terminer en avril 2010. Ils visent à la réalisation d'un chenal de navigation (12 mètres de profondeur, $120 \mathrm{~m}$ de large, 4,5 km de long) entre le port industriel associé à l'usine et la passe du Duroc qui permet la sortie en haute mer, au-delà du récif-barrière. Les études préliminaires ont estimé à $7300000 \mathrm{~m}^{3}$ le volume des déblais à extraire dans des matériaux, variables selon les zones, depuis des silts, des boues et des sables jusqu'à des constructions coralligènes indurées.

Ces caractéristiques ont nécessité la mise en œuvre sur le terrain de deux types de dragues sur les 13 zones caractérisées par les travaux de reconnaissance (figure 1). Il a été utilisé, d'une part, une DAET (drague autoporteuse à élinde traînante) d'une capacité de $3700 \mathrm{~m}^{3}$, et d'autre part, une DE (drague excavatrice dotée d'un godet de pelle de $40 \mathrm{~m}^{3}$ ), assistée de deux barges autopropulsées de $3700 \mathrm{~m}^{3}$ chacune. La DAET a été affectée au dragage des sédiments fins meubles et la DE à celui des sédiments consolidés.

Le matériel dragué sur la zone littorale pour atteindre la bathymétrie de projet est transporté vers la zone d’immersion des déblais, située à l'extérieur du récif-barrière, sur des fonds supérieurs à $1000 \mathrm{~m}$. Le site d’immersion des déblais (suspension concentrée pour la DAET et sédiment semi-solide saturé en eau pour les barges associées à la $\mathrm{DE}$ ) est un carré de $1 \mathrm{~km}$ d'arête, situé à une distance de $5 \mathrm{~km}$ de la pente externe du récif-barrière, au sud-ouest de la passe de Duroc. Sa localisation, réputée sans incidence sur la biodiversité récifale, a fait l’objet de nombreuses modélisations 
validées par INERIS, tiers-expert de l'étude d'impact environnementale générale du Projet Koniambo. Les conclusions du tiers expert, rendues en 2005, ont permis la prise de l'arrêté n ${ }^{\circ}$ 180/2005 du 31/12/2005 portant autorisation domaniale de réaliser des travaux de dragage, d'endigage et d'immersion des déblais sur le domaine public maritime de la province Nord.

Un PGE (plan de gestion environnementale) de l'impact des travaux a été demandé par INERIS dans ses recommandations. Compte tenu du spectre large des aspects traités par le PGE, la communication cite de façon très succincte les caractéristiques générales de ce suivi environnemental et focalise sur un seul enjeu : la turbidité ambiante.

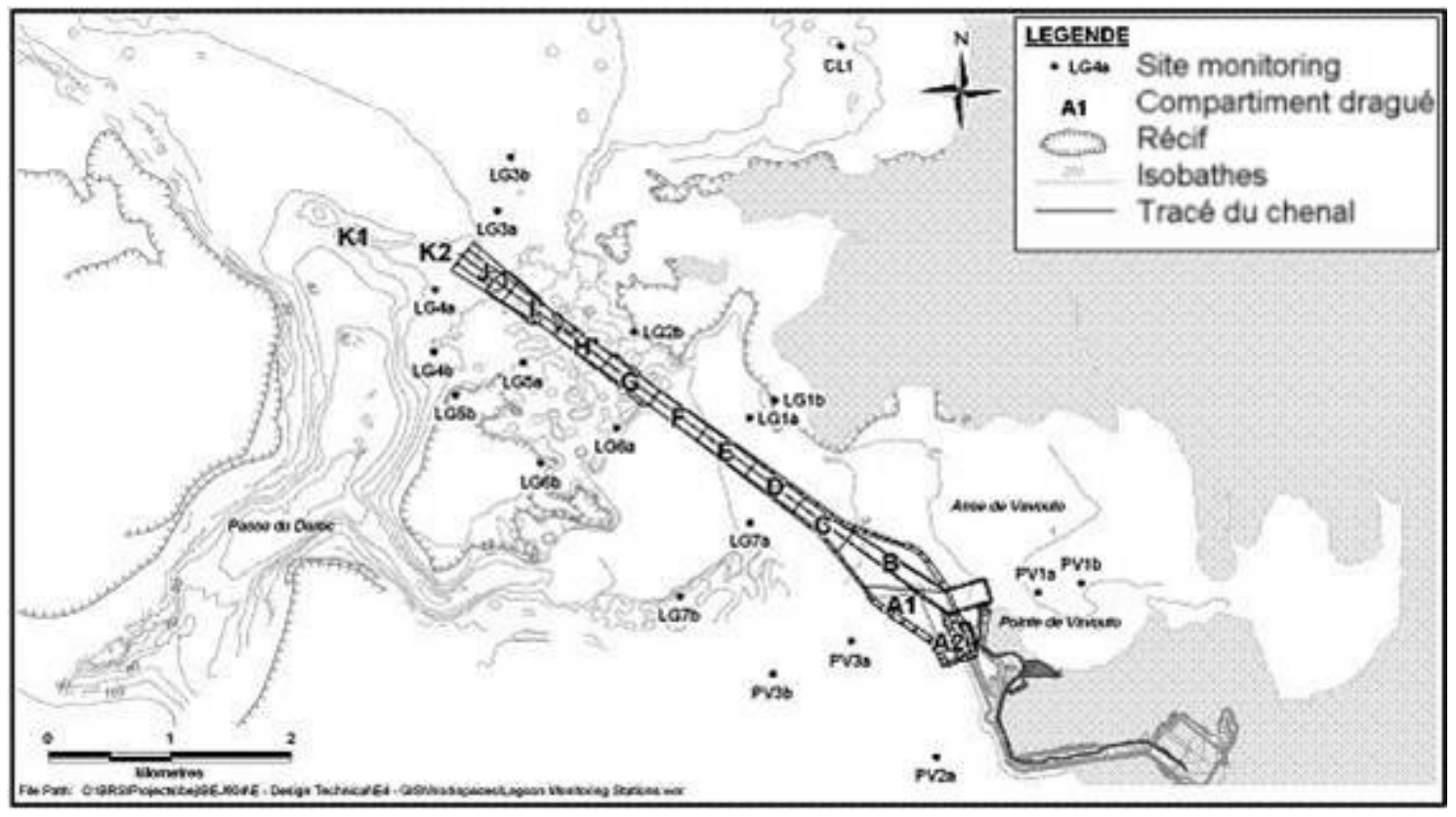

Figure 1. Le tracé du chenal et les 13 zones de travail (d'après KBR-Rapport BEJ604).

\subsection{Un contexte environnemental sensible}

Les travaux intéressent un domaine lagonaire situé à proximité immédiate de zones récemment classées (2008) au patrimoine mondial de l’humanité.

Réalisée à 85\% aujourd'hui, l'avancée des travaux permet de dégager une analyse critique sur le degré d'efficacité du programme de suivi environnemental mis en place en regard des attentes exprimées. Le projet industriel de KNS (Koniambo Nickel Society) est, en effet, au centre d'un pari essentiel pour le pays : celui du développement durable à réussir dans le respect d'un environnement fragile et des pratiques sociétales des populations autochtones qui vivent dans la zone impactée.

Le fort attachement culturel des mélanésiens envers leur milieu naturel les rend très attentifs au respect de l'environnement. Un important clan pêcheur (tribu d'Oundjo) de la région VKP est, en effet, installé à proximité immédiate de l'emprise foncière du site industriel de Vavouto; il a manifesté son inquiétude pour les travaux maritimes qui 
impactent ses lieux de pêche. Les contraintes sur l'industriel en matière de respect de l'environnement sont donc fortes du côté des populations locales.

Elles sont également fortes du côté des associations et organisations internationales qui œuvrent sur l'environnement. Le classement du récif calédonien impose des contrôles stricts et des pratiques exemplaires en vue de la sauvegarde des sites naturels. La réalisation d'un chantier important a généré des questionnements logiques sur les risques encourus par l'écosystème. Le peu d'échos négatifs qui se sont fait jour jusqu'ici, au sujet du chantier du dragage, démontre le succès de l'approche suivie en terme d'information : politique de communication claire, partenariat attractif avec les populations locales, création du comité environnemental du Koniambo.

\section{Le plan de suivi environnemental du dragage de Vavouto (volet turbidité)}

\subsection{Généralités sur la démarche suivie}

A la fin 2007, le plan de gestion environnementale pour le dragage du chenal, demandé par l'INERIS en 2005, a été produit par Kellog Brown Root Ltd (KBR, 2007) pour le compte de KNS et présenté à la province nord. Celle-ci a mandaté un tiers-expert, l'université de la Nouvelle Calédonie (UNC), pour le valider et après remise des conclusions de ce dernier (ALLENBACH, 2008), les travaux ont débuté en août 2008. Le plan distingue 6 enjeux d'importance variable : la qualité de l'eau, de l'air, l'écologie (flore et faune), le bruit, la gestion des déchets et des substances dangereuses.

\subsection{Les mesures relatives au contrôle de la turbidité ambiante à l'intérieur du lagon}

Seul le volet contrôle de la turbidité ambiante à l'intérieur du lagon sera détaillé dans le cadre de la communication. Il s'agit, d'une part, de l'effet induit le plus apparent, susceptible non sans raison, de fédérer les craintes et les oppositions aux travaux et d'autre part, du paramètre le plus pénalisant pour l'écosystème corallien.

Le tableau 1 permet de comparer la proposition initiale produite par KBR pour le compte de KNS et les mesures retenues par l'autorité provinciale après la tierceexpertise UNC. Le système de bouées fixes mis en place pour mesurer la turbidité en temps réel (radio-transmission des données recueillies, au pas d'une mesure/3 mn) et de façon plus classique (stations fixes autonomes travaillant au pas d'une mesure/30 mn, déchargées hebdomadairement) est constitué par 2 bouées OMC-045-B-1200 (mesure de la turbidité et de la houle) et 8 bouées OMC-45-B-250 (mesure de la turbidité). Les bouées ont été équipées de sondes multi-paramètres de type YSI 6920 et HYDROLAB MS ET DS 5. Le travail de terrain est assuré par deux prestataires sous la supervision de HT (Hatch-Technip) et KBR. 


\subsection{Les résultats du suivi}

Le suivi intra-lagonaire, autour de l'emprise directe du chenal, a respecté le plan d'échantillonnage préconisé et les capteurs ont été déployés très rapidement. A contrario, au-delà du récif, sur la pente externe, les capteurs ont été posés plus tardivement (8 mois après le début du chantier) et une partie de l'information a été perdue. Le traitement des 18 premiers mois de données montre qu'il n’y a pas eu de dépassement significatif des seuils autorisés (> à 70 NTU sur 1 heure ou > à 30 NTU sur 3 jours). Les seuls dépassements notés sont liés à des problèmes de salissure (biofooling) et à des pannes de capteurs, solutionnés par des interventions de nettoyage des plongeurs et des équipes de maintenance. Des dysfonctionnements (signal haché) ont également été notés pour les données transmises par radio, résolus par le déplacement des relais.

Tableau 1. Comparatif des mesures préconisées et retenues.

\begin{tabular}{ll}
\hline Mesures proposées par KNS & Mesures mises en place \\
\hline Surveillance visuelle journalière des & Mise en place d'un système de bouées \\
panaches sédimentaires ; & d'acquisition et de transmission en temps \\
Mesures toutes les 30 minutes de la & réel (pas de 3 minutes) des valeurs de \\
turbidité de l'eau et déchargement & turbidité de l'eau ; \\
hebdomadaire des données & Mesures en parallèle, toutes les 30 minutes \\
enregistrées par bouées spécifiques. & de la turbidité de l'eau et déchargement \\
& hebdomadaire des données ; \\
& Mesures directes manuelles à proximité de \\
& la zone de dragage ; \\
& Mise en place d'un système d'alerte se \\
& déclenchant en cas de dépassement des \\
& valeurs critiques retenues par le projet. \\
\hline
\end{tabular}

\subsection{La communication environnementale}

L'autorité provinciale et l'industriel se sont accordés pour instituer une cellule d'interface entre la population et le projet Koniambo : le CEK. Composée d'élus, de scientifiques, de représentants du monde associatif et coutumier, directement intégrée dans le projet qui la subventionne, elle est animée par des techniciens environnementalistes, salariés par elle. Elle intervient efficacement pour le respect des engagements pris ou pour l'obtention de compensations environnementales en cas de dysfonctionnements. Elle anime les séances de restitution des résultats du suivi, crée les espaces de discussion souhaitables. La démarche est incontestablement un succès puisqu'au bout de 18 mois et à moins de 3 mois de son terme, le programme ne souffre d'aucune critique significative. 


\section{Organisation des données}

Dans le cadre du PGE du dragage, la quantité de données disponibles est importante : environ 5 Go de données sur le seul volet de la turbidité, généralement sous forme tableurs ou de documents texte: mesures de turbidité, images satellites de la zone impactée, rapports d'investigations sur les profils de profondeur et les panaches selon le type de bateau, rapports de surveillance visuelle du panache, rapports mensuels de l'activité des bateaux, rapports de maintenance des sondes, comptes-rendus d'audits des bateaux, localisation des déversements.

Face à la quantité de données produites, on pouvait légitimement espérer une structuration claire de ces données, d'ailleurs demandée par la tierce-expertise ayant conduit à l'autorisation de dragage. Au dépouillement, il s'avère qu'il s'agit d'un amas de données sans réelle organisation. Les mesures ont été effectuées par des sociétés différentes et apparaissent disparates. Elles ont menées suivant différents protocoles, en fonction des sociétés impliquées et des capteurs utilisés. Elles ont été restituées dans des formats hétérogènes, non structurés dans une base de données interopérable. Le manque d'organisation de ces données hétérogènes, tant sur plan de la structure que sur "le fond", constitue un obstacle majeur pour une vision globale des phénomènes à observer et elle a rendu plus difficiles, d'une part, la tâche de l'expert en charge du contrôle des travaux et d'autre part, la communication vers les décideurs et populations.

Au plan du contrôle, cette carence de vision d'ensemble, en amont du projet, a contraint à une réorganisation complète des données acquises. Celle-ci a nécessité l'identification des paramètres communs à chaque type de mesure et de leurs spécificités, afin de les intégrer dans une base de données cohérente et exploitable. Ce travail fastidieux, coûteux en temps et ressources humaines, a consisté à faire ce que l'on pourrait appeler de la "rétro ingénierie", à partir des données disponibles et des rapports remis pour tenter de comprendre la démarche adoptée par les différents acteurs et leurs propres contraintes :

- Qu'est-ce qui a été mesuré ?

- Qui a fait la mesure ?

- Quand a été faite la mesure ?

- Où a été faite la mesure ?

- Comment a été faite la mesure ?

- Dans quelles conditions a été faite la mesure (direction/vitesse du vent, direction/hauteur de houle, les cycles des marées) ?

- Quel est le résultat de la mesure ?

L’organisation de cette "rétro base de données" et des métadonnées associées est naturellement passée par la définition d'un modèle conceptuel permettant de définir clairement les relations existantes entre les diverses séries de données. Outre les données de turbidité disponibles et ce, pour des besoins d'analyse, des données 
supplémentaires ont été intégrées dans la base, telles que par exemple les valeurs seuils de turbidité définies dans le PGE, le passage des dragues à proximité des stations de mesures, pour pouvoir répondre aux questions de l'expert (applications-métier). Parmicelles-ci, il a été rendu possible d'extraire des mesures effectuées sur une période donnée, à une station précise ou sur une zone plus large, l'accès aux paramètres de calibration des sondes utilisées, aux sorties cartographiques des résultats, etc.

La structure de la base a été réalisée, en respectant les standards ouverts afin d'assurer l'interopérabilité, à l'aide de PowerAMC qui permet de réaliser un modèle conceptuel de données (MCD). Les données ont été intégrées en suivant cette structure à une base POSTGRES.

\section{5. Élaboration d'un outil d'analyse des séries de turbidité}

L'accès aux données étant maintenant facilité par la mise en place d'une base de données, il s'est agi de développer les outils capables de répondre aux besoins de l'expertise du suivi des données environnementales.

Le premier besoin exprimé consistait à analyser efficacement les données de turbidité issues des différents capteurs sur une période déterminée, afin de croiser avec d'autres paramètres comme le travail d'une drague ou le passage d'une barge ou d'un bateau, un événement climatique de forte intensité ou simplement la détection d'une avarie de capteur. Il s'agissait donc de produire une synthèse spatiale et temporelle des données de turbidité sous la forme de cartes interpolées pour des périodes quotidiennes, hebdomadaires, mensuelles ou annuelles. Cela permet, en particulier, de comparer aisément l'état initial d'une zone avant, pendant et après les travaux de dragage et d'étudier la résilience du milieu.

Un autre besoin exprimé était de pouvoir détecter automatiquement les zones et les périodes au sein desquelles des valeurs seuils ont été dépassées et de les visualiser cartographiquement.

Un prototype a été réalisé pour répondre aux questions de l'expert. Il interroge la base de données, effectue des traitements statistiques et permet de présenter les résultats des analyses. De manière pratique, plusieurs cartes sont produites et permettent de visualiser les valeurs de turbidité, selon que les mesures ont été collectées ponctuellement sur site ou en continu via des capteurs avec un relevé transmis par télémétrie ou non. Une carte supplémentaire présente l'ensemble des mesures. Selon le souhait de l'expert, il est ainsi possible de discriminer les types de mesures et de les comparer. Quatre cartes peuvent être produites pour la période choisie et ce, pour chaque profondeur où l'interpolation est possible.

Une courbe de l'évolution temporelle de la turbidité (aux stations et profondeurs choisies) peut également être réalisée, afin d’étudier la nature du dépassement (pic ponctuel, par exemple). Il a été démontré que la variable de turbidité possède bien toutes les caractéristiques statistiques d'une "variable régionalisée" (DROESBEKE et al., 
2006) et que l'interpolation des valeurs de la turbidité sur l'ensemble de la zone de dragage à partir d'un nombre de points (6 stations a minima) est représentative de la zone observée. La méthode d'interpolation choisie est le krigeage, issu de la géostatistique et qui permet d'appréhender la structure spatiale du phénomène étudié.

L'analyse de la structure spatiale s'effectue par le calcul d'un variogramme expérimental qui mesure le degré de dissimilarité entre les points en fonction de leur éloignement (variance). Il caractérise la continuité spatiale et la régularité du phénomène. Un variogramme modèle est ajusté au variogramme expérimental (Marcotte) afin d'automatiser le processus d'interpolation. Une échelle fixe de couleurs a été choisie afin de faciliter la comparaison des cartes et d'avoir un meilleur rendu de l'évolution de la turbidité au cours des travaux.

Un exemple de courbe temporelle est présenté figure 3. Cette courbe a été produite automatiquement pour mettre en évidence un pic de turbidité observé le 7 avril 2004, à une profondeur comprise entre 1 et $2 \mathrm{~m}$. Il est intéressant de noter que le pic détecté à la station LG3b se retrouve également dans l'enregistrement des stations voisines LG4b, LG5b et PV3b et durant la même période. Au vu des résultats, l'expert peut conclure que ces dépassements de seuil sont la conséquence d’un même événement qui s'est propagé dans la zone et peut décider d'approfondir la recherche afin de découvrir la cause de cette anomalie.

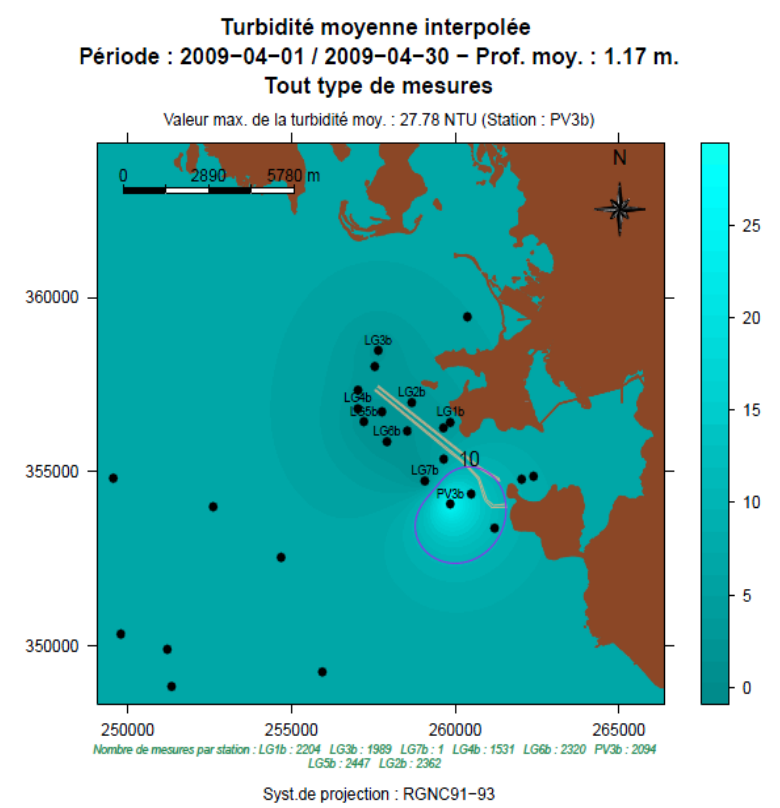

(Le contour bleu indique une zone où la valeur de turbidité a dépassé le seuil autorisé)

Figure 2. Exemple de distribution spatialisée de la turbidité. 


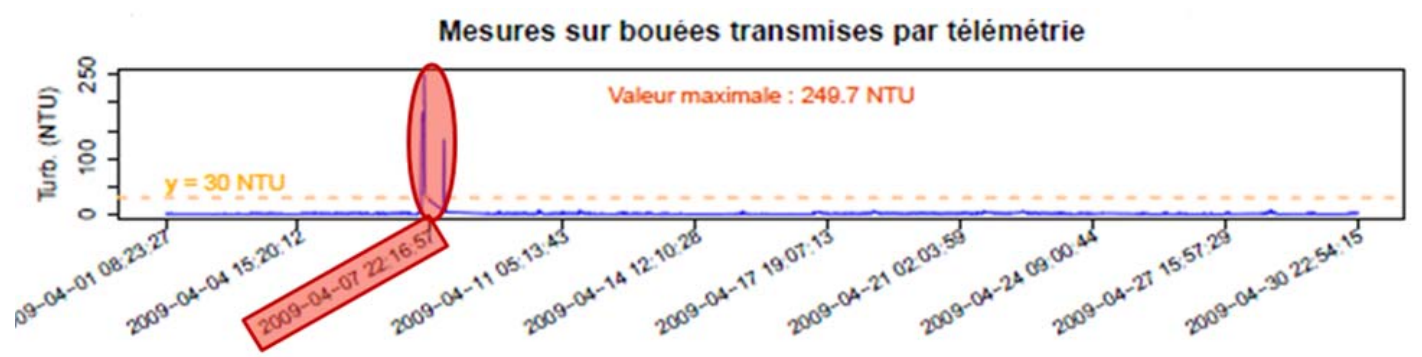

Figure 3. Evolution temporelle de la turbidité à la station LG3b, entre $1 \mathrm{~m}$ et $2 \mathrm{~m}$, en avril 2009.

\section{Améliorations potentielles de la démarche et conclusion}

L'analyse et la transmission des résultats auraient pu être facilitées si le recueil, l'organisation, le stockage et la diffusion de la donnée avaient été mieux organisés. Une réflexion associant l'ensemble des acteurs (bureaux d'étude, compagnie minière, experts et collectivités), menée en amont des travaux, aurait donné une plus grande cohérence dans le processus allant de l'acquisition à l'analyse. Il ne s'agit pas, ici, de tenter d'uniformiser les productions de chaque acteur, sachant que chacun possède ses outils et sa manière de fonctionner, mais plutôt de fournir des recommandations afin que ces productions puissent s'insérer dans un système d'information directement exploitable à des fins d'analyse et de visualisation d'indices, voire d'outils d'aide à la décision.

\subsection{L’intérêt de la création d'une base de données interopérables}

Diverses initiatives, tant européennes (INSPIRE) qu'internationales, (GMES, GEOSS) visent à fédérer les activités sur les systèmes d'information afin d'optimiser l'utilisation des infrastructures existantes et futures. L’objectif est de développer les moyens de collecte et de diffusion de données et de permettre leur intégration dans des projets de suivis environnementaux. L'évolution des Systèmes d'Informations Environnementaux (SIE) s’appuie sur des architectures distribuées et interopérables. L'intérêt de telles approches réside dans la facilité de partage et de diffusion des informations à différentes communautés d'acteurs (décideurs, services techniques, secteur privé et grand public). Parallèlement, des initiatives de standardisation ont vu le jour pour favoriser: (a) l'utilisation d'applications ouvertes pour le traitement de l'information provenant des capteurs, (b) la coopération entre les fournisseurs de systèmes de capteurs, leurs gestionnaires et les "consommateurs" des données.

Plusieurs organisations fournissent des systèmes d'acquisition de données, mais peu d'entre elles fournissent des interfaces et aucune n'offre des outils génériques normalisés. Aussi, l’Open GIS Consortium (OGC) s'est donné pour objectif de publier des spécifications pour la structuration de la diffusion des données issues de capteurs de tous types hébergés et contrôlables par Internet. L'objectif des normes est de permettre la mise en place de réseaux de capteurs interrogeables à distance selon des protocoles 
publics. Les standards de la suite "Sensor Web Enablement" (SWE) proposés par l'OGC constituent un cadre riche pour normaliser l'acquisition, le stockage et la diffusion des informations, concernant aussi bien la description des capteurs eux-mêmes que les données issues de ces capteurs. À titre d'exemple, les spécifications suivantes font partie de cette suite :

- le langage "Sensor Markup Language" permet de définir dans un format commun la description des capteurs et des systèmes de capteurs (géolocalisation, traitement des observations, programmation, abonnements à des alertes, etc.).

- la spécification "Observations and Measurements" (O\&M) définit un modèle conceptuel de données pour organiser les bases de données (relatives aux observations d'événements situés temporellement) et mesures réalisées (valeur correspondant à un phénomène observé). En dehors de ces deux valeurs, O\&M permet également de décrire d'autres propriétés comme le processus qui a permis d'obtenir ces valeurs, la position spatiale (primordiale pour des capteurs mobiles), la qualité de la mesure.

Il est intéressant de noter que, même pour les capteurs qui ne peuvent pas transmettre les mesures et être pilotés à distance selon les standards préconisés, il est possible d'alimenter une base de données "tampon" qui permet de simuler une partie des comportements d'un capteur standardisé. La conformité à ces standards facilite également le croisement des données issues des capteurs réels ou simulés avec d'autres standards de l'OGC largement répandus et dédiés à l'information géographique, tels que "Web Map Service" (WMS) et "Web Coverage Service" (WCS).

En s’affranchissant des difficultés liées à la disparité des capteurs et en se basant sur des modèles de données reconnus, l'élaboration et l'utilisation d'un système d'information sont grandement simplifiées. Il est à souligner que cette étape est cruciale et souvent négligée ou sous-estimée ; ce qui conduit à un surcoût de travail par la suite, à une quantité ou qualité de données inadéquates aux besoins, et in fine, à des difficultés quelquefois insurmontables pour analyser les données. Ce système d'information sert de socle pour développer, de manière générique, les outils de diffusion/visualisation mais également pour fournir des tableaux de bord thématiques synthétiques à des fins d'alerte, de concertation et de décision.

\subsection{L’intérêt d'un traitement optimisé de données hétérogènes naturalistes}

Dans l'étude de Vavouto, les données de turbidité étaient fournies sous forme de tableaux permettant d'opérer des statistiques de base, afin d'en estimer la qualité, la fiabilité et la précision (détermination des moments d'ordre 1 et supérieur, fréquence, tendance et saisonnalité, estimation du bruit et détection des données aberrantes et/ou manquantes). Un travail de nettoyage et de vérification de la donnée a cependant été nécessaire. L'automatisation du processus était rendue difficile par l'éparpillement des données dans plusieurs fichiers ou supports multi-formats. 
L’organisation des informations récoltées dans une base de données structurée, adossée à un protocole déterminé et enrichi par des métadonnées associées suffisamment détaillées pour déterminer la provenance et les conditions de recueils aurait grandement simplifié cette nécessaire phase d'analyse exploratoire. Ceci est essentiel dans le cadre d'études environnementales qui intègrent des données de nature hétérogène (quantitative continue ou discrète, qualitative ordonnée ou non...) et de qualité variable.

Outre la qualification des données, un des objectifs fixés était de déterminer les valeurs de turbidité dépassant un seuil déterminé et de croiser ces évènements avec d'autres facteurs, tels que le nettoyage des capteurs, par exemple. La possibilité d'exploiter une base de données structurée permettant d'effectuer des requêtes temporelles et spatiales croisées apparaissait ici particulièrement intéressante et aurait entraîné un gain de temps substantiel, si elle avait été faite au départ plutôt qu'a posteriori et à des fins de contrôle. Il est à noter, en effet, que la plupart des logiciels de statistiques évolués tels que $R$ (DRAY \& DUFOUR, 2007) autorisent les requêtes sur les bases de données les plus courantes.

L’expérience de la base de données "turbidité" de Vavouto permet de dégager quelques recommandations d'ordre général pour le projet Koniambo et au-delà de celui-ci.

La mise en œuvre du triplet base de données/outils d'analyse/système d'information est d'ores et déjà conseillée à l'industriel et aux décideurs politiques dans le cadre du plan de suivi environnemental général du projet Koniambo, qui se poursuit au-delà des opérations de dragage. Dans ce type de démarche, il s'agit d'évaluer les corrélations entre les variables afin de calculer des interpolations et de modéliser leurs dynamiques spatio-temporelles. A partir des modélisations, des indicateurs peuvent être construits. Ces indicateurs doivent être élaborés par les experts et les intervalles de confiance, les biais, et les précisions associées fournis. La dynamique des données peut être étudiée, soit en mode univarié (dynamique d'une variable particulière telle que la turbidité dans notre cas), soit en mode multivarié (i.e. estimer la distribution spatiale et temporelle des sédiments en fonction de données de courantologie, météorologiques et autres). Cette étape nécessite l'utilisation de modèles mathématiques qui peuvent s'avérer complexes à mettre en œuvre (définition de la structure du modèle et calibrage des paramètres). A partir des indicateurs et des sorties de modèle, des recommandations automatiques peuvent être proposées afin d'aider les décideurs sur les mesures à prendre.

Ces outils peuvent s'appuyer sur des méthodologies allant d'une combinaison linéaire simple des paramètres déterminants jusqu'à un processus complexe pouvant faire intervenir des concepts d'intelligence artificielle tels que les réseaux bayésiens (BORGELT \& KRUSE, 2002). Ces graphes probabilisés possèdent notamment des capacités permettant de prendre en considération simultanément les connaissances a priori des experts (à travers une structure de graphe) et l'information contenue dans les données. 
7.12 : Revue Paralia - Vol. 3 (2010)

\section{Références bibliographiques}

ALLENBACH M. (2008). Projet Koniambo (travaux de dragage) - Analyse critique du plan de suivi environnemental. Rapport, Université de la Nouvelle-Calédonie, 69 p.

BORGELT C., KRUSE R. (2002). Graphical Models: Methods for Data Analysis and Mining. Chichester, UK: Wiley. ISBN 0-470-84337-3.

DROESBEKE J.-J., LEJEUNE M., SAPORTA G. (2006). Analyse statistique des données spatiales. Paris: Technip.

DRAY S., DUFOUR A.B. (2007). The ade4 package: implementing the duality diagram for ecologists. Journal of Statistical Software 22(4), pp 1-20.

KBR -Kellog Brown Root Ltd- (2007). Plan de suivi environnemental des travaux de dragage, Rapport BEJ604-G-REP-001, 47 p. 